\title{
COMPACT SEMIGROUPS IRREDUCIBLY CONNECTED BETWEEN TWO IDEMPOTENTS ${ }^{1}$
}

\author{
W. M. FAUCETT
}

The simplest and most familiar compact connected space which can be provided with the structure of a (topological) group is the simple closed curve $C$ (topological circle). Perhaps the most natural example of a compact connected (topological) semigroup is the closed unit interval $I$ with the usual multiplication. The space $C$ admits the unique structure of a group, whereas simple examples show (see Examples 1, 2,3) that the space $I$ admits many structures of a semigroup. Such multiplications need not be abelian, may admit both nilpotents and idempotents, and may not have a zero element.

In this note we initiate the analysis of the semigroup structures with which the space $I$ may be provided. While our theorems are much more general, the following corollary will give a fair picture of our results. Suppose that $I$ admits such a multiplication that its end points play the natural roles of zero and unit. If there are no other idempotents and no nilpotent elements except zero, then the multiplication must be that of the real numbers. The major step in the proof is that of showing that unique $q$ th roots exist for any positive dyadic rational $q$.

It is with pleasure that we acknowledge the helpful suggestions and advice of A. D. Wallace in the preparation of this paper.

We define a mob to be a Hausdorff space together with a continuous, associative multiplication. A clan is a compact connected mob with unit. If $S$ is a mob, a set $T \subset S$ is a left (right) ideal if $T \neq \square$ and $S T \subset T(T S \subset T)$. A two-sided ideal is both a left and right ideal. Using Clifford's terminology [1], we shall denote the minimal twosided ideal of a mob $S$ by $K$ and the set of idempotents of $S$ by $E$. By a zero element, we mean an element 0 , such that $0 x=0=x 0$ for all $x \in S$. We define an element $s$ to be nilpotent if $s^{n}=0$, for some positive integer $n$.

A connected space $S$ is irreducibly connected between two points $a$ and $b$ if no proper connected subset of $S$ contains both $a$ and $b$. In such a space, every point different from $a$ and $b$ is a cut point, separating the space into exactly two components [6]. We can intro-

Presented to the Society, November 28, 1953, under the title $A$ result on mobs irreducibly connected between two points; received by the editors July 30,1954 and, in revised form, December 6, 1954.

1 This work was done under Contract N7-onr-434, Task Order III, Navy Department, Office of Naval Research. 
duce a linear order relation in $S$ by defining $y<x$ if $y \in C, C$ the component of $b$ in $S \backslash\{x\}$. This order induces a topology in $S$. Since we wish to use the order topology in this paper, we observe that if $S$ is compact the order topology is equivalent to the original topology of $S$. Consider

$$
f:(S, U) \rightarrow(S, V)
$$

where $U$ is the original topology of $S, V$ the order topology and $f$ the identity function. Since $V \subset U$ and $S$ is compact, $f$ is a homeomorphism, establishing our assertion that the two topologies are equivalent. In this paper if zero is a non-cut point, we shall define the order relation in $S$ so that $0 \leqq x, x \in S$. We shall also denote

$$
[s, t]=\{y: y \in S, s \leqq y \leqq t\} .
$$

The set $[s, t]$ is compact and by an easy argument is seen to be irreducibly connected between $s$ and $t$.

Lemma 1. Let $S$ be a compact connected mob and $t \in S \backslash K, K$ the minimal two-sided ideal of $S$. If $S \backslash\{t\}=A \cup B, A \mid B, K \subset A$, then St, $t S$ and $S t S \subset A^{*}$.

Proof. Let $J$ be the union of all left ideals of $S$ contained in $A$. Since $K \subset A, J \neq \square$ and $J$ is a left ideal. Since $J$ is open [3] and $S$ is connected, it follows that $t \in J^{*}$. Since $J^{*}$ is an ideal, $S t \subset S J^{*} \subset J^{*}$ $\subset A^{*}$. Similarly $t S \subset A^{*}$ and $S t S \subset A^{*}$.

We observe that this lemma gives a new proof of the result that if $S$ is a clan no element of $H(u)$, the maximal subgroup containing $u$, the unit of $S$, cuts $S[5]$.

LEMMA 2. If $S$ is a clan with zero and $S$ is irreducibly connected between $u$ and 0 , then $S x=x S=[0, x]$ for all $x \in S$.

Proof. Obviously $[0, x] \subset S x$ and equality follows from Lemma 1.

It is of interest to remark that if we define $y \leqq x$ if and only if $y S \subset x S$ and $S y \subset S x$, then by virtue of Lemma 2 this order relation, derived from the algebraic structure of $S$, coincides with the order relation derived from the topological structure of $S$.

Lemma 3. Let $S$ be a clan with zero and let $S$ have no idempotents other than zero and unit. If $S$ is irreducibly connected between $u$ and 0 , then $S$ is abelian. Further, $S$ contains a countable dense set and is a homeomorph of the unit interval of real numbers.

Proof. Let $\Delta^{n}$ be the diagonal of the Cartesian product of $S n$ times. For each $n$ define $f_{n}: \Delta^{n} \rightarrow S$ by $f_{n}(y)=x^{n}$ where $x$ is the projection of $y$ into $S$. Since $f_{n}$ is continuous, $f_{n}\left(\Delta^{n}\right) \subset S$ is a connected set 
containing $u$ and 0 and is therefore equal to $S$. This implies that $f_{n}$ is onto and in particular, that any $x \in S$ has an $n$th root.

We now assert that if $x \neq 0$, then $x$ has a unique square root. Suppose there exist elements $c$ and $d$, such that $c^{2}=d^{2}=x$. We may assume $d<c$ and, by Lemma 2, $d=a c=c b$ for some $a, b \in S, a, b \neq u$. We now have $c^{2} b=x b \leqq x$. We claim $x b=x$, otherwise we would have a contradiction to Lemma 2 since $a(x b)=a c^{2} b=d^{2}=x$. But $x b=x$ implies that $x \in \cap_{n=1}^{\infty} S b^{n}=S e$ where $e \in E$ and $e \in \Gamma(b)=\left\{b, b^{2}, b^{3}, \cdots\right\}^{*}$ $[2 ; 4]$. However since $b \neq u$, it follows from Lemma 1 that the only idempotent in $\Gamma(b)$ is 0 which is a contradiction. This establishes, that for $x \neq 0, x$ has an unique square root, and by induction that $x$ has unique $2^{m}$ th roots, where $m$ is any positive integer.

Let us define for $x \in S, x \neq 0$,

$$
\left(x^{p / 2^{q}}\right)=\left(x^{1 / 2^{q}}\right)^{p}
$$

$p$ and $q$ being positive integers. Straightforward verification establishes that we can now write

$$
x^{r} x^{s}=x^{r+s}
$$

where $r$ and $s$ are positive dyadic rationals.

For $x \in S, x \neq 0, u$, let

$$
D=\left\{x^{r}: r \text { a positive dyadic rational }\right\} .
$$

$D$ is an abelian submob by (1.1) and we claim that $D^{*}=S$. That $D$ meets every open neighborhood of 0 follows from the fact that $0 \in \Gamma(x)$. If $V$ is a basis element in the order topology containing $u$ and if $y \in V, y \neq u$, then $y^{2^{n}}<x$ for some positive integer $n$. We can easily verify that $x^{1 / 2^{n}} \in V$.

Let $t$ be an arbitrary element of $S, t \neq 0, u$, and let $B=\{s \mid a<s<b\}$ be an arbitrary basis element in the order topology containing $t$. Without any loss of generality we can assume $0<a<b<u$. Let

$$
\begin{aligned}
R^{\prime} & =\left\{r \mid x^{r} \geqq b, r \text { a positive dyadic rational }\right\}, \\
R^{\prime \prime} & =\left\{r \mid x^{r} \leqq a, r \text { a positive dyadic rational }\right\} .
\end{aligned}
$$

$R^{\prime}$ and $R^{\prime \prime}$ are nonempty and have upper and lower bounds respectively. Let $r^{\prime}$ be the l.u.b. of $R^{\prime}$ and $r^{\prime \prime}$ the g.l.b. of $R^{\prime \prime}$. Clearly $r^{\prime} \leqq r^{\prime \prime}$ otherwise we would have an immediate contradiction to Lemma 2. If $r^{\prime}<r^{\prime \prime}, D$ meets $B$. Therefore assume $r^{\prime}=r^{\prime \prime}$. Let $\left\{p_{n}\right\}$ be a monotone increasing sequence of dyadic rationals converging to $r^{\prime}$. The sequence $\left\{x^{p_{n}}\right\}$ is monotone decreasing in $S$ and since $S$ is compact, clusters at some point $c \geqq b$. But in view of Lemma 2, $\left\{x^{p_{n}}\right\}$ converges to $c$. Let $W$ be an open set, $c \in W$, such that $W \cap[0, a]$ 
$=\square$. By the continuity of multiplication, there exist open sets $U$ and $V, u \in U, c \in V$, such that $U V \subset W$. But $D \cap U \neq \square$; therefore $x^{r} \in U$ for some $r$. There exists a $p_{k}$ in the sequence $\left\{p_{n}\right\}$ such that $x^{p_{k}} \in V$ and $r+p_{k}>r^{\prime}=r^{\prime \prime}$. This implies that $x^{r} x^{p_{k}}=x^{r+p_{k}} \in[0, a]$, which is a contradiction. It follows that $D^{*}=S$ and that $S$ is abelian.

Since $D$ is a countable dense set and $S$ is compact, $S$ is homeomorphic to $I$, the unit interval of real numbers [6].

THEOREM 1. If $S$ is a compact mob irreducibly connected between two idempotents $f$ and $g$ and contains no other idempotents, then $S$ is abelian and homeomorphic to $I$.

Proof. Since $S$ is compact, $S$ has a minimal two-sided ideal $K$, $K=\bigcup\{H(e): e \in E \cap K\}$, where $H(e)$ is the maximal subgroup containing $e[1]$. $S$, a compact connected linearly ordered space, certainly has the fixed point property. Since $H(e)=e S e$ is a retract of $S$, $H(e)$ has the fixed point property and consequently $H(e)=e$. Hence every element in $K$ is idempotent. Since $K$ is connected, $K=f$ or $K=g$. If $K=f, f$ is a zero for $S$ and $g$ is a unit since $g S=S g=S$. The conclusion follows from Lemma 3.

Theorem 2. Let $S$ satisfy the hypotheses of Theorem 1 and in addition let $S$ contain no nilpotent elements. Then there exists a function $f$ from $S$ to I that is an isomorphism as well as an order-preserving homeomorphism.

Proof. Fix $x \in S, x \neq 0, u$, and let

$$
D=\left\{x^{r}: r \text { a positive dyadic rational }\right\} .
$$

As shown in Lemma 3 and Theorem 1, D is a countable dense set in $S$. Furthermore we assert we have the relation

(1.2) $\quad x^{r}<x^{8} \quad$ if $s<r, s$ and $r$ positive dyadic rationals.

By Lemma 2 and (1.1) in Lemma 3, we have $x^{r} \leqq x^{8}$ if $s<r$. Suppose $x^{r}=x^{s}$ and $r=s+t$ for some $t, t$ a dyadic rational. By (1.1) we have $x^{r}=x^{s+t}=x^{s} x^{t}=x^{s}$. Therefore $x^{s} \in \bigcap_{n=1}^{\infty} S\left(x^{t}\right)^{n}=S e$ where $e \in \Gamma\left(x^{t}\right)$ $[2 ; 4]$. But by the same type of argument used in the proof of Lemma $3, e=0$, which implies that $x^{s}=0$, a contradiction since $S$ contains no nilpotent elements.

We define $g: D \rightarrow I$ by $g\left(x^{r}\right)=(1 / 2)^{r}$. We define $f: S \rightarrow I$ by $f(u)=1$ and, for $s \neq u, f(s)=$ g.l.b. $\left\{g\left(x^{r}\right): x^{r} \geqq s\right\}$. Clearly $f \mid D=g$; that is, $f\left(x^{r}\right)=g\left(x^{r}\right)=(1 / 2)^{r}$. From (1.2) and the fact that $D$ is dense in $S$, we have 


$$
a, b \in S, \quad a<b \text { implies } f(a)<f(b) .
$$

It is immediate from this relationship that $f$ is an order-preserving homeomorphism.

It remains to be proved that $f$ is a homomorphism. For $a, b \in S$ we claim $f(a b)=f(a) f(b)$. Suppose $f(a b)>f(a) f(b)$. This implies there exist $r^{\prime}$ and $r^{\prime \prime}$, dyadic rationals, such that $(1 / 2)^{r^{\prime}} \geqq f(a)$ and $(1 / 2)^{r^{\prime \prime}}$ $\geqq f(b)$ and $f(a b)>(1 / 2)^{r^{\prime}+r^{\prime \prime}}$. But $x^{r^{\prime}} \geqq a$ and $x^{r^{\prime \prime}} \geqq b$ implies that $x^{r^{\prime}+r^{\prime \prime}} \geqq a b$, whence, by (1.3), $f(a b) \leqq f\left(x^{r^{\prime}+r^{\prime \prime}}\right)=(1 / 2)^{r^{\prime}+r^{\prime \prime}}$, a contradiction. On the other hand assume $f(a b)<f(a) f(b)$. There exists a dyadic rational $t$ such that $t>t^{\prime}+t^{\prime \prime}$ and $f(a b)<(1 / 2)^{t}<f(a) f(b)$, where

$$
\begin{aligned}
t^{\prime} & =\text { l.u.b. }\left\{r: r \text { a dyadic rational, } x^{r} \geqq a\right\}, \\
t^{\prime \prime} & =\text { l.u.b. }\left\{r: r \text { a dyadic rational, } x^{r} \geqq b\right\} .
\end{aligned}
$$

By (1.3), $x^{t}>a b$. By the continuity of multiplication in $S$ and the fact that $D$ is dense, there exist $r^{\prime}$ and $r^{\prime \prime}$, such that $x^{r^{\prime}} \geqq a$ and $x^{r^{\prime \prime}} \geqq b$ and $x^{t}>x^{r^{\prime}+r^{\prime \prime}} \geqq a b$. But this is a contradiction to (1.2) since by the choice of $t, t>r^{\prime}+r^{\prime \prime}$. This completes the proof that $f(a b)$ $=f(a) f(b)$.

Lemma 4. Let $S$ be a compact mob with zero and let $S$ be irreducibly connected between two points. If $S^{\prime}=[f, e]$, where e and $f$ are idempotents, and if $f$ acts as a zero on $S^{\prime}$, then $S^{\prime}$ is a submob.

Proof. Let

$$
\begin{aligned}
M & =\{s: s \in S, e<s\}, \\
N & =\{s: s \in S, s<f\} .
\end{aligned}
$$

Clearly if $0 \neq f, 0 \in N$. By Lemma 1 we have $S^{\prime} x \cap M=\square$ for all $x \in S^{\prime}$. Suppose $S^{\prime} x \cap N \neq \square$ for some $x \in S^{\prime}$. Consequently there exists $y \in S^{\prime}$ such that $y x \in N$. Let $D=[x, e]$. Since $e$ acts as a unit on $S^{\prime}, y \in y D$. Therefore there exists $d \in D$, with $x<d$, such that $y d=f$. But $x=d t, t \in S^{\prime}$, and we have $y x=y d t=f t=f$, a contradiction. This establishes that $S^{\prime}$ is a submob.

Lemma 5. Let $S$ be a clan with zero. If $S$ is irreducibly connected between $u$ and 0 , then $S$ is abelian.

Proof. Let $x$ be an arbitrary element of $S$. If $x \in E$, the set of idempotents of $S$, then for $y<x$, we have $y \in S x=x S$ and $y x=x y=y$. On the other hand, if $x<y$, then $x=y a=b y$ for some $a, b \in S$. We assert $x y=x$, otherwise $x=x^{2}=x(y a)=(x y) a \leqq x y<x$. Similarly we show $y x=x$. 
If $x \notin E$, let

$$
\begin{aligned}
T^{\prime} & =[x, u] \cap E, \\
T^{\prime \prime} & =[0, x] \cap E .
\end{aligned}
$$

$T^{\prime}$ and $T^{\prime \prime}$ are non-null compact linearly ordered subsets of $S$ and certainly have maximal and minimal elements. Let $e$ be the minimal element of $T^{\prime}$ and $f$ the maximal element of $T^{\prime \prime}$. If $S^{\prime}=[f, e], S^{\prime}$ is a compact abelian submob by Lemmas 3 and 4 . Hence $x$ commutes with $y$ if $y \in S^{\prime}$. As we have already observed for $y>e, y e=e y=e$, and for $y<f, y f=f y=y$. Therefore we have $y x=y e x=e x=x=x e=x e y=x y$ if $y>e$. On the other hand if $y<f, y x=y f x=y f=y=f y=x f y=x y$.

Theorem 3. Let $S$ be a clan. If $S$ is irreducibly connected between two idempotents, then $S$ is abelian if and only if $S$ has a zero.

Proof. Assume $S$ has a zero. By Lemma 1, the unit of $S$ is one of the two non-cut points of $S$. If the other idempotent is the zero element of $S, S$ is abelian by Lemma 5 . Otherwise let $S^{\prime}=[0, u]$ and $S^{\prime \prime}=[e, 0]$ where $e$ is the other non-cut point of $S . S^{\prime}$ is a compact abelian submob by Lemmas 4 and 5 . A slight modification of the method of proof used in Lemma 4 establishes that $e S^{\prime}=S^{\prime} e=S^{\prime \prime}$. To complete the proof it is sufficient to show that $e$ commutes with every element of $S^{\prime}$. This is immediate since $x e=e x e=e x$ for all $x \in S^{\prime}$.

Conversely, noting that $S$ has the fixed point property, we consider the sets, $P(s)=\{x \mid x s=x\}, s \in S$. The collection $\mathbb{P}=\{P(s) \mid s \in S\}$ is a collection of non-null closed sets. Since $S$ is abelian, it is easily verified that $P$ has the finite intersection property. Therefore $\cap P \neq \square$, which establishes that $S$ has a zero. As an alternate proof, we recall the observation made in the proof of Theorem 1 that if $S$ has the fixed point property, the maximal subgroups in $K$ are single elements. The known result that $K$ is a group if $K$ meets the center of $S$ [2] implies that $K$ is trivial, therefore a zero.

TheOREM $4 .^{2}$ If $S$ is a compact connected mob irreducibly connected between two idempotents $e$ and $f$, then $S$ is abelian if and only if $S$ has a zero and ef $=f e$.

Proof. If $S$ is abelian, $S$ has a zero by the same argument as in Theorem 3 and obviously $e f=f e$.

To establish the sufficiency, assume $e f=f e$ and that $S$ has a zero. If either $e$ or $f$ is the zero element of $S$, the other is obviously a unit

2 The author is indebted to the referee for suggestions improving the proof of Theorem 4. 
for $S$ and the result follows by Theorem 3. Otherwise, let $S^{\prime}=[0, e]$ and $S^{\prime \prime}=[f, 0] . S^{\prime}$ and $S^{\prime \prime}$ are abelian submobs by Lemmas 4 and 5 . Suppose ef $\in S^{\prime \prime}$. Let $T=[e f, e]$. By Lemma 1, $T$ is an ideal, and it follows that $T=S e=e S$. Since $e f \in E, T$ is abelian by Theorem 3. To prove the theorem it suffices to show $x<e f$ and $t \in T$ imply $x t=t x$. Now $x t=(x f)(e t)=(x e f) t$, and $x e f \in T$ by Lemma 1 . Hence $(x e f) t$ $=t(x e f)=t(x f) e=t x e=(t e)(x e)=t(e x) e=t e(e x)=(t e) x=t x$. This completes the proof.

Example 1 (E. CAlabi). Let $S$ be the set of all real numbers $x$ such that $1 / 2 \leqq x \leqq 1$. For $x, y \in S$ define the product of $x$ and $y$ to be the $\max (1 / 2, x y) . S$ is a clan with zero and every element in $S$ other than the unit is nilpotent. $S$ is homeomorphic with the Euclidean straight line interval $I$ but is clearly not algebraically isomorphic with $I$.

Example 2. Consider the following set

$$
S=\left\{\left(\begin{array}{ll}
x & 0 \\
0 & 1
\end{array}\right)\right\} \cup\left\{\left(\begin{array}{ll}
0 & y \\
0 & 1
\end{array}\right)\right\}, \quad 0 \leqq x \leqq 1,0 \leqq y \leqq 1
$$

$S$ is a clan irreducibly connected between two idempotents that commute. But $S$ does not have a zero and $S$ is not abelian.

EXAMPLE 3. Consider the following set

$$
S=\left\{\left(\begin{array}{ll}
x & 0 \\
x & 0
\end{array}\right)\right\} \cup\left\{\left(\begin{array}{ll}
0 & y \\
0 & y
\end{array}\right)\right\}, \quad 0 \leqq x \leqq 1,0 \leqq y \leqq 1 .
$$

$S$ is a compact connected mob with zero and is irreducibly connected between two idempotents that do not commute. Hence $S$ is not abelian.

Examples 2 and 3 show that the sufficiency conditions cannot be eliminated in Theorems 3 and 4.

\section{REFERENCES}

1. A. H. Clifford, Semigroups containing minimal ideals, Amer. J. Math. vol. 70 (1948) pp. 521-526.

2. R. J. Koch, On topological semigroups, Tulane University dissertation, 1953.

3. R. J. Koch and A. D. Wallace, Maximal ideals in compact semigroups, Duke Math. J. vol. 21 (1954) pp. 681-686.

4. K. Numakura, On bicompact semigroups, Mathematical Journal of Okayama University vol. 1 (1952) pp. 98-108.

5. A. D. Wallace, $A$ note on mobs, Anais da Brasileira de Ciencias vol. 24 (1952) pp. 329-334.

6. R. L. Wilder, Topology of manifolds, Amer. Math. Soc. Colloquium Publications, vol. 32, 1949, pp. 21-39.

The Tulane University of Louisiana 